\title{
Comunicação em Saúde Pública na Prevenção do Consumo Excessivo de Álcool e Drogas na População Escolar de São Tomé e Príncipe: Protocolo Científico
}

\author{
Prevention Harmful Consumption of Alcohol and \\ Drugs in Sao Tome and Principe Through Public Health \\ Communication: The Scientific Protocol
}

\author{
Isabel DE SANTIAGO $\rrbracket^{1}$, Leonor Bacelar NICOLAU ${ }^{2}$, Rui Tato MARINHO ${ }^{3,4}$, José PEREIRA-MIGUEL ${ }^{5}$ \\ Acta Med Port 2020 Apr;33(4):229-236 - https://doi.org/10.20344/amp.13435
}

\section{RESUMO}

Introdução: São Tomé e Príncipe é um País Africano de Língua Oficial Portuguesa, de rendimento baixo-médio, onde a extrema pobreza potencia grandes desigualdades em saúde. Pouco se sabe sobre o consumo de álcool e substâncias ilícitas, além dos indicadores de importação e distribuição pela população. Não existem estudos realizados sobre este tema em crianças e adolescentes e são desconhecidas quaisquer medidas preventivas. Além disso, as bases de dados manuais apresentam limitações significativas, perante a inexistência de causas associadas às taxas de mortalidade ( 0 - 5 anos e > 5), e incapacidade de estabelecer uma relação causa-efeito entre doenças e morte e esperança média de vida. Inexistência de dados relativo às causas de mortalidade nos relatórios do Centro Nacional de Endemias ou nos relatórios da organização não-governamental Instituto Marquês de Valle Flor, entidade facilitadora de cuidados de saúde em especialidades clínicas selecionadas em regime de voluntariado de médicos de hospitais Portugueses. Propusemos assim realizar o primeiro diagnóstico de situação familiar e condições de habitação, e sobretudo, sobre o consumo de álcool e de substâncias ilícitas nos jovens. Neste sentido, foi desenvolvido o Inquérito Nacional sobre o Consumo de Álcool e Drogas em Meio Escolar em São Tomé e Príncipe para melhor caraterização da situação na população escolar, e para testar estratégias de comunicação em saúde e intervenções preventivas adaptadas às realidades socioculturais das populações-alvo. Foram considerados o dialeto, as famílias monoparentais, (estrutura matriarcal) a poligamia (sobretudo nos homens) num país e com governos liderados por homens (estrutura patriarcal), onde o papel da mulher, como refere a Organização das Nações Unidas para a Alimentação e a Agricultura, continua esquecido. Posteriormente procederemos à recolha de amostras de álcoois tradicionais, nas duas principais ilhas, para análise (pelo Laboratório de Estudos Farmacêuticos e Laboratório Nacional de Engenharia Civil) e determinar os metais pesados no processo de produção e impacto na esperança média de vida.

Material e Métodos: Revisão da literatura nas bases de dados eletrónicas da Organização Mundial da Saúde, Organização Mundial da Saúde África, Organização das Nações Unidas, SCOPUS, MEDLINE, The Lancet e Lancet Global Health. Também considerámos e analisámos os dados disponíveis no Instituto Nacional de Estatística, Ministério das Finanças, Estatísticas da Educação, Ministério da Educação, Cultura e Formação, Estatística da Saúde, Ministério da Saúde e Assuntos Sociais do XV Governo de Iniciativa Presidencial 2013-14. Serão realizadas algumas entrevistas qualitativas aos líderes de todas as comunidades, à igreja e missões católicas com intervenção humanitária e apoio aos mais desfavorecidos. Posteriormente, aplicação de um questionário biográfico, demográfico e socioeconómico sobre o uso de substâncias lícitas e ilícitas a uma amostra de 2064 estudantes. Foram consideradas as caraterísticas demográficas, sociais e culturais. Após resultados e conclusões, iremos ensaiar algumas intervenções preventivas de comunicação em saúde como o edutainment nas escolas primárias de três distritos (EDUCA_TARTARUGA) e com os jornalistas de rádio (EDUCA_PRESS), as quais serão avaliadas pelos professores primários e pelos jornalistas de rádio.

Palavras-chave: Comportamento do Adolescente; Comunicação-em-Saúde; Consumidores de Droga; Consumo de Bebidas Alcoólicas; Drogas llícitas; Estilo de Vida; Estudantes; Ilhas Atlânticas

\section{ABSTRACT}

Introduction: Sao Tome and Principe is an African low-and-middle-income country, where extreme poverty causes major health inequalities. No systematic research has been done on the consumption of alcohol and drugs in Sao Tome and Principe, and only overall statistics are available based on the importation of alcoholic drinks and their distribution among the population. There are also no studies on consumption of alcohol and illicit substances in children and youth and no preventive measures being undertaken. Besides that manual databases present significant limitations, considering the lack of causes associated with mortality rates $(0-5$ years and $>5)$, and the difficulty to establish a cause/effect relation between diseases, deaths and life expectancy. No relevant data with burden of life was found in the reports of Centro Nacional de Endemias or the non-governmental, organization Instituto Marques de Valle Flor, a facilitator on healthcare clinical specialties selected on a voluntary basis by doctors from Portuguese hospitals. So, we proposed to provide a first overview of family and housing conditions, and above all, the consumption of alcohol and illegal drugs in young people. Thus, a project, the National Survey on Harmful Consumption of Alcohol and Drugs in Schools of Sao Tome and Principe, will be realized in order to better characterize the situation among children and young students and test public health communication strategies and preventive interventions aimed at this target-population. Interventions were designed taking into consideration local sociocultural realities

1. Comunicação em Saúde Pública. Instituto de Medicina Preventiva e Saúde Pública. Faculdade de Medicina. Universidade de Lisboa. Lisboa. Portugal.

2. Economia da Saúde. Instituto de Medicina Preventiva e Saúde Pública. Faculdade de Medicina. Universidade de Lisboa. Lisboa. Portugal.

3. Serviço de Gastrenterologia e Hepatologia. Hospital Universitário de Santa Maria. Centro Hospitalar Universitário de Lisboa Norte. Lisboa. Portugal.

4. Department of Gastroenterology and Hepatology. Faculdade de Medicina. Universidade de Lisboa. Lisboa. Portugal.

5. Instituto de Medicina Preventiva e Saúde Pública. Faculdade de Medicina. Universidade de Lisboa. Lisboa. Portugal.

$\square$ Autor correspondente: Isabel De Santiago. isabeldesantiago@medicina.ulisboa.pt

Recebido: 14 de janeiro de 2020 - Aceite: 10 de fevereiro de 2020 | Copyright @ Ordem dos Médicos 2020 
of target audiences. We considered dialect language, single-parent families (matriarchal structure) and polygamy (mostly) in men and a country and governments led by men (patriarchal structure) and, in which the woman's role, as Food and Agriculture Organization of the United Nations reports, remains overlooked. Subsequently, we will collect traditional alcohols samples from the two main islands for analysis (at Laboratório de Estudos Farmacêuticos and Laboratório Nacional de Engenharia Civil - Portugal) and to determine heavy metals in the production process and impact on burden of life.

Material and Methods: In order to characterise the country's situation in terms of alcohol and illicit substances consumption a literature review was carried out through a search in several international electronic databases, such as those of the World Health Organization, World Health Organization Africa, United Nation, The Lancet and Lancet Global Health, etc. Available data of the following institutions of Sao Tome and Príncipe was also analyzed: National Institute of Statistics, Ministry of Education, Culture and Training and Ministry of Health and Social Affairs. Several interviews with community and church leaders as well as with members of catholic missions were carried out to better understand the local situation. Following this, a nationwide cross-sectional survey of a sample of 2064 students will be carried out. This will include a questionnaire on socio-demographic characteristics, lifestyles, health behaviors/attitudes, alcohol and illicit substances consumption. Finally, based on the overall diagnosis obtained, some edutainment health communication preventive interventions will be tested in the primary schools of three districts (EDUCA_TURTLE) and on the radio journalists (EDUCA_PRESS). These were evaluated by primary school teachers and by radio journalists.

Keywords: Alcohol Drinking; Atlantic Islands; Drug Users; Health Communication; Illicit Drugs; Life Style; Students

\section{INTRODUÇÃO}

São Tomé e Príncipe (STP) é um pequeno arquipélago, com duas ilhas, geográfica e estrategicamente posicionado no Golfo da Guiné, e integra o grupo de Países Africanos de Língua Oficial Portuguesa (PALOP). Trata-se de um low and medium income country [(LMIC), país de rendimento baixo-médio] e é o segundo mais pequeno país africano. Em STP observamos um elevado nível de pobreza, que é potenciadora de grandes desigualdades em saúde..$^{1-3}$ O país apresenta uma realidade social ${ }^{1}$ e epidemiológica ${ }^{4}$ caracterizada pelos baixos níveis ${ }^{5}$ de estilos de vida saudáveis ${ }^{4,6}$ educação formal e política de comunicação em saúde.$^{6}$ Os indicadores gerais disponíveis baseiam-se na importação de bebidas alcoólicas, ${ }^{7,8}$ a principal do país $(60 \%)$.

O Global Status Report on Alcohol and Health da Organização Mundial de Saúde (OMS) aponta que o consumo de álcool e drogas, ${ }^{8}$ e seu uso excessivo, ${ }^{9}$ é causador de vários tipos de doenças. Segundo a OMS, o uso de álcool causa 3,3 milhões de mortes em todo o mundo. ${ }^{8} \mathrm{O}$ consumo excessivo de álcool (CEA), substância oncogénica, constitui um fator de risco de cancro. ${ }^{10,11} \mathrm{O}$ consumo excessivo de drogas também é causa de outras doenças não transmissíveis (DNT), ${ }^{12,13}$ como transtornos mentais e comportamentais, ${ }^{14}$ sendo também responsável por traumas, ${ }^{7,15}$ acidentes de viação, anos de vida perdidos e morte. ${ }^{16,17}$ Trata-se de uma séria ameaça à saúde pública. ${ }^{18-20}$

Pouco se sabe, com rigor, sobre o consumo excessivo $(C E)^{9,21}$ de álcool e drogas (CEAD) nos PALOP. ${ }^{20}$ Todavia, existem inúmeros alertas trazidos a público com reportagens de televisão e rádio e na imprensa escrita, de que pode tratar-se, de facto, de um sério problema de saúde pública, ${ }^{22-24}$ agravado pelos determinantes sociais. ${ }^{25}$ É essa a visão da OMS África, ${ }^{13,26}$ que tem feito diversos alertas sobre esta problemática que se crê que tem vindo a aumentar. ${ }^{27} \mathrm{~A}$ situação tende a piorar, atendendo ao facto de que as crianças e os jovens ${ }^{18,28}$ seguem os padrões comportamentais de gerações mais velhas, tanto na produção artesanal como no consumo. Estes dois fatores têm levado ao agravamento da situação global de saúde. ${ }^{28} \mathrm{Na}$ região da África lusófona, em algumas populações, como é o caso de Moçambique, foram registados episódios de binge drinking ${ }^{29}$ que caraterizam consumos excessivos em adultos ${ }^{29}$ Este problema de Saúde Pública ameaça as populações mais vulneráveis e pobres, como as de Africa, ${ }^{30}$ mas no caso de STP, com um índice muito elevado de pobreza no relatório UNICEF, ${ }^{31}$ incide especialmente nas crianças e jovens. ${ }^{18,32,33}$ Tendo em conta a existência de estudos ou indicadores sobre esta problemática, torna-se urgente a necessidade de investigar para capacitar e equilibrar desigualdades. ${ }^{34} \mathrm{Em}$ 2014, a OMS estimou o consumo anual de álcool importado per capita (APC) em maiores de 15 anos, em 8,7 litros (2003 - 2005) e 7,1 litros (2008 - 2010), não contabilizando os álcoois artesanais, já que a população mais pobre ${ }^{20,31}$ não tem acesso a esses consumos (importados). No entanto, não existem quaisquer indicadores relativamente a jovens ${ }^{10,35}$ ou população escolar, ou sobre os efeitos do álcool nas DNT ou nas mortes em STP. Além desta, não existe informação nacional ou evidência científica sobre tipos, quantidades produzidas ou consumidas de álcoois artesanais, nem de drogas. Sem indicadores reais na população escolar, na literatura sobre África e STP, os indicadores existentes são o STEPS wise approach to Surveillance (STEPS), sobre doenças crónicas (DC) na população com mais de 50 anos e o Multiple Indicator Cluster Survey (MICS). No caso de STP, os padrões de consumo são desconhecidos ${ }^{36}$ e os consumos de álcool ${ }^{9,37,38}$ estendem-se aos aguardentes artesanais como o grogue (originário dos descendentes da comunidade de Cabo Verde) e a cacharamba (originários dos descendentes da comunidade de Angola e STP), sendo que no caso de STP se verifica a utilização de catalisadores (fermentação como referido popularmente) e baterias (baterias alcalinas com metais). Em STP, não era possível avaliar as quantidades ingeridas de álcool devido à inexistência de evidência científica sobre todos os tipos de álcoois artesanais produzidos ${ }^{39} \mathrm{sem}$ controle sanitário ou legislação $0^{40,41}$ e consumidos nas comunidades locais. ${ }^{39,40,42}$ As formas mais comuns de álcool são cacharamba, chalelinha, tomatxo e quentekú, bebidas produzidas a partir de frutos como cana-de-açúcar, manga, cajamanga ou abacaxi, e catalisadas com substâncias ${ }^{40,42}$ (metais desconhecidos), excluindo o vinho de palma com processo de fermentação natural. Por outro lado, o controlo sobre algumas substâncias ilícitas (cannabis) que podem ser de fácil acesso e consumo é limitado. As crianças 
(através da observação de padrões de consumos nos pais e irmãos) e os jovens são a população mais vulnerável $\left.\right|^{9,21,43,44}$ ao desenvolvimento de hábitos de CE de álcool, ${ }^{25,45-48} \mathrm{com}$ importantes implicações para a saúde ao longo da vida. O risco de dependência e de adição futura é muito elevado se este consumo excessivo começar antes dos 15 anos. ${ }^{21}$ Além disso, algumas intervenções preventivas como comunicação em saúde ${ }^{49}$ (IPCS) através do edutainment, ${ }^{50}$ que visem a promoção da saúde ${ }^{45}$ e redução de risco causado pelos CE de álcool e drogas (CEAD), ${ }^{45,46}$ são recomendadas para as populações mais jovens. ${ }^{41,45}$ Trata-se de uma prioridade, nestas ilhas africanas do Atlântico, promovendo a educação para a saúde, ${ }^{45,47}$ procurando infletir os encargos com a saúde. O CEAD em África e em STP é um importante determinante de saúde, que contribui também para o aumento das desigualdades sociais. ${ }^{51}$ É neste contexto que, considerado um dos grupos mais vulneráveis, crianças e jovens (ou a população escolar), ${ }^{46,47}$ se determinou a urgente necessidade de realizar um estudo, que designámos de INCEAD STP - Inquérito Nacional sobre o Consumo de Álcool e Drogas em Meio Escolar em São Tomé e Príncipe (Fig. 1).

Trata-se de uma investigação pioneira, urgente, considerando a sobrecarga (BURDEN) e Disability-Adjusted Life Years (DALY) potenciados pelos CEAD, na medida em que os estudos existentes sobre AD não incluem populações escolares da África Lusófona, nem de STP. Com base nos resultados a apurar e nessa evidência, avaliar-se-á a necessidade de pilotar modelos de intervenções preventivas de comunicação em saúde ${ }^{47,48,49}$ que visem a promoção de saúde e modelos de salutogénese ${ }^{49,50}$ com posterior avaliação. Também neste domínio, apesar da literatura apontar a necessidade de realizar ${ }^{7}$ intervenções preventivas, nos PALOP são escassos ou inexistentes e colocam dificuldades particulares. As intervenções preventivas nos países em desenvolvimento devem conter caraterísticas especiais, que obrigam a observação e conhecimento dos comportamentos das comunidades envolvidas ${ }^{52}$ a literacia

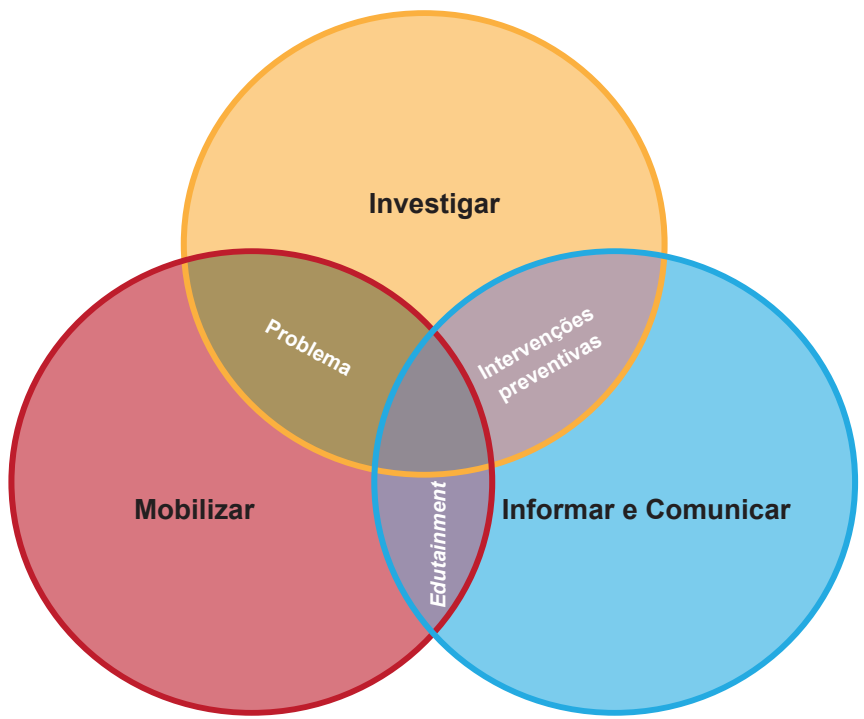

e tradições das populações alvo, valorizando relações sociais e saúde. Neste sentido, é indispensável a adaptação da evidência das IPCS à realidade STP, através do uso dos dialetos de conversação dialetu e crioulo, utilizados em sessões de educação para a saúde de configuração especial designadas de edutainment, ${ }^{50}$ com e para as comunidades. ${ }^{53}$ Esta metodologia de comunicação em saúde traduz-se na conjugação de dois conceitos de saúde pública: Educação e Entretenimento, ${ }^{50,54,55}$ Concluindo, a problemática do consumo de substâncias lícitas e ilícitas em STP existe e é necessário aprofundar com rigor científico o seu conhecimento, de modo a melhor intervir, recorrendo a práticas e metodologias de promoção da saúde e mobilização social da população alvo ${ }^{51-53}$ e instrumentos de edutainment. ${ }^{50,54}$ Finalmente, consideramos uma etapa determinante para a melhoria as condições de vida destas populações, proceder à recolha de amostras dos álcoois tradicionais, produzidos no seio das comunidades, nas duas principais ilhas, e vendidos e consumidos pela população nacional e estrangeiros, para análise e determinar os metais pesados no processo de produção e impacto no BURDEN.

De acordo com os pontos mencionados anteriormente, os objetivos gerais do estudo são: 1) Realização de um inquérito nacional que determine a frequência e a distribuição do consumo de álcool e drogas na população escolar jovem em São Tomé e Príncipe. 2) Identificação das principais caraterísticas associadas aos consumos excessivos suscetíveis de intervenções preventivas. 3) A partir dos resultados obtidos, propor e ensaiar intervenções preventivas usando metodologias de Comunicação em Saúde Pública, nomeadamente de edutainment. ${ }^{50,54}$

\section{MATERIAL E MÉTODOS \\ Diagnóstico da situação}

O diagnóstico preliminar de situação inclui a revisão da literatura e o Inquérito Nacional sobre o Consumo de Álcool e Drogas em Meio Escolar em São Tomé e Príncipe

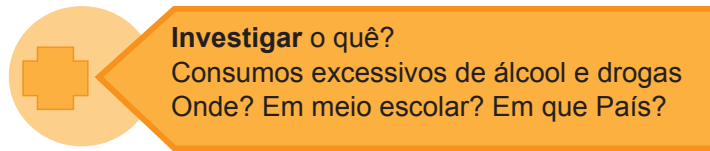

Investigar o quê?

Consumos excessivos de álcool e drogas Onde? Em meio escolar? Em que País?
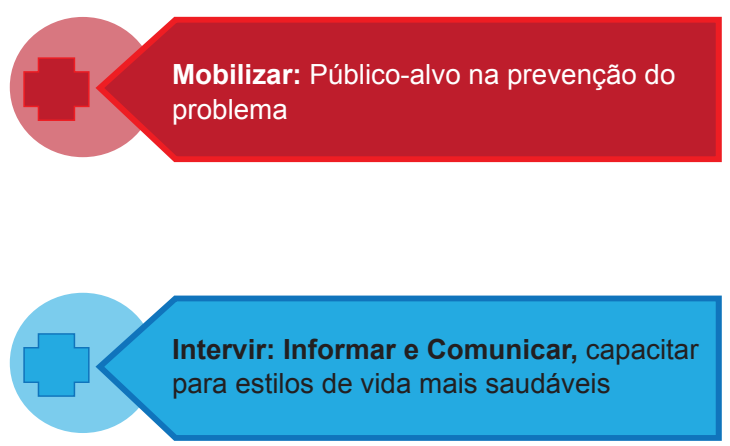

Figura 1 - Modelo de intervenções preventivas de Comunicação em Saúde Pública na prevenção de doenças: Investigar, Mobilizar, Comunicar 


\section{(INCEAD STP).}

\section{Recolha de dados estatísticos nacionais}

Com vista à concretização do estudo, entende-se indispensável a análise de dados estatísticos, recolhidos junto das instituições ligadas aos sectores da educação, da saúde e das finanças de São Tomé e Príncipe. Esta etapa do trabalho de investigação passa pelo contato direto com o Gabinete de Planeamento e Inovação Educativa - Departamento de Estatística, do Ministério da Educação, Cultura e Formação, no que se refere às estatísticas da Educação 2012 do Ministério da Educação, Cultura e Formação, bem como dados estatísticos CENSOS 2012, obtidos junto do Instituto Nacional de Estatística (INE), tutelado pelo Ministério das Finanças.

\section{Identificação de serviços de apoio e autorizações ofi- ciais}

Para a realização do presente estudo identificaram-se diferentes serviços ligados aos sectores de educação, saúde, justiça e educação. A aprovação de todas as autoridades relevantes de STP, incluindo do Ministério da Educação, Cultura e Formação e da Direção Nacional de Ensino Secundário, foi obtida antes da aplicação do questionário. Além disso, o estudo foi aprovado pelo Comissão de Ética do Centro Hospitalar de Lisboa Norte, Faculdade de Medicina da Universidade de Lisboa e Centro Académico de Medicina de Lisboa, CAML, número de referência 47/16.

\section{Reconhecimento de comportamentos socioculturais}

Com vista à concretização do estudo, entende-se indispensável a audição de várias entidades ligadas ao sector social e religioso, pela sua ação de solidariedade relevante junto de diferentes públicos-alvo. Estas ações sociais são realizadas por instituições como a Santa Casa de Misericórdia de São Tomé e Príncipe (SCMSTP). Com base neste processo de observação, foi feita uma análise dos documentos e contributos dos diferentes dirigentes, através de entrevistas e da realização de reuniões de trabalho com entidades oficiais para compreender motivos conducentes à dependência de álcool. Do ponto de vista sociocultural, realizaram-se entrevistas para conhecer as motivações associadas ao tráfico de droga e seu consumo.

\section{Método \\ Inquérito nacional}

Realização do inquérito nacional, designado: "A Comunicação em Saúde na prevenção do consumo excessivo de álcool e drogas em São Tomé e Príncipe" através da aplicação de questionários nas escolas de todos os distritos do país a nível do Primeiro e Segundo Ciclos do ensino secundário, do ensino profissional noturno e alfabetização, do ensino técnico e do ensino superior.

\section{População-alvo}

Desenho da amostra, população e critérios de inclusão. Será realizado um inquérito-piloto com recolha de dados no Liceu Nacional de São Tomé com uma amostra de conveniência de 150 indivíduos do sexo masculino e feminino. A aplicação dos questionários-piloto conta com o apoio do Ministério da Educação, Cultura e Formação e da Direção Nacional do Ensino Secundário. Constituem critérios de inclusão ter entre 13 e 18 anos de idade (na amostra de conveniência do Liceu Nacional de São Tomé), diagnóstico assumido (auto reportado) como dependente ou consumidor de substâncias, estabelecido há seis meses, e concordar em participar no estudo. O questionário será adaptado à linguagem utilizada em STP - português de conversação santomense -, um português diferente do comummente falado em Portugal no que se refere ao tipo de vocabulário e seu sentido. Com base neste inquérito piloto, foi possível refinar e objetivar as perguntas do questionário a aplicar no estudo. A população-alvo do estudo INCEAD STP corresponde aos alunos inscritos e a frequentar o ensino público, e compreendia 16924 alunos no ano letivo de 2014. A amostra final de 2064 inquiridos, correspondendo a $12 \%$ da população alvo, permite um erro máximo de $2 \%$ para um nível de confiança de $95 \%$ na estimativa de proporções relevantes. $O$ inquérito foi aplicado em escolas secundárias, ensino qualificante profissionalizante (diurno e noturno) e ensino superior públicos dos sete distritos do país. Utilizou-se um método aleatório de turmas escolhidas pela direção de cada escola, em que o género masculino e feminino esteja proporcionalmente representado. As turmas apuradas para o inquérito nacional, são as com maior número de alunos, numa escala também proporcional com os demais liceus e escolas do país e habitantes.

\section{Questionário}

O questionário, ajustado à linguagem utilizada em STP, português de conversação santomense, diferente do comummente falado em Portugal, é constituído por 121 questões, distribuídas em cinco partes, que se passam a especificar: Parte I, Perguntas 1 a 28: Dados biográficos e sociodemográficos; Parte II, Perguntas 29 a 58: Uso de substâncias lícitas e ilícitas (álcool e drogas); Parte III, Perguntas 59 a 68: Saúde e a situação da família; Parte IV, Perguntas 69 a 72: Uso não médico por parte de membros da família, de medicamentos; Parte V, Perguntas 73 a 121. Saúde do inquirido.

\section{Recolha e tratamento de dados}

$\mathrm{Na}$ recolha de dados, aplicar-se-á um questionário adaptado com o modelo aplicado nos inquéritos levados a cabo pela equipa do investigador Casimiro Balsa. ${ }^{55,56}$ Pretende-se integrar neste estudo questões que vão ao encontro de necessidades de informação dessas instituições, potenciando deste modo a utilidade dos resultados do estudo. Depois de os questionários estarem preenchidos, serão digitalizados e registados por leitura ótica automática pela Unidade de Epidemiologia da Unidade de Acolhimento da Investigação no Instituto de Medicina Preventiva e Saúde Pública, Faculdade de Medicina, Universidade de Lisboa. Posteriormente serão aplicados procedimentos de 
controlo de qualidade e de validação da base de dados, de modo a assegurar que não existem enviesamentos que invalidem os resultados apurados. Está contemplada ainda uma análise da representatividade da amostra a nível da variável de estratificação de distrito, mas também por sexo e idade. Os resultados serão apurados para a amostra total e cruzados pelas principais variáveis de caracterização e de estudo: distrito, sexo, idade, escolaridade, tipo de droga consumida, padrão de consumo e estado de saúde (sintomas). Serão construídas tabelas de frequências (frequências absolutas e percentagens) e de cruzamento (frequências e percentagens por coluna), complementadas por análise inferencial com aplicação de testes de hipóteses adequados. Complementarmente, prevemos o recurso e aplicação de métodos de estatística multivariada como análise de clusters, de modo a identificar perfis de consumo e de sintomatologia. Os resultados a apresentar decorrem das análises estatísticas de dados e comparações entre os grupos a realizar pelo teste do qui-quadrado ou teste exato de Fisher se necessário. Como responder a cada pergunta não é obrigatório (opcional), algumas das variáveis podem não ter respostas completas para os 2064 participantes. As análises vão ser realizadas no programa IBM SPSS. E consideramos um nível de significância de $\alpha=0,05$.

\section{Ética}

Todos os dados serão tratados de forma confidencial e só estarão disponíveis para a equipa de investigação e para os objetivos do projeto. Os inquéritos são anónimos e todos os participantes serão informados sobre os objetivos gerais do estudo. Os alunos das diferentes turmas, escolas e ISP (atual Universidade de São Tomé e Príncipe), dos sete distritos (incluindo ilha do Príncipe) serão informados em detalhe sobre os objetivos do estudo, a anonimização do questionário, e sobre a não obrigatoriedade de resposta total ou parcial. Desta forma, asseguraremos que o preenchimento do questionário anónimo corresponderá ao consentimento informado do inquirido. Os dados a recolher manualmente serão submetidos a leitura ótica pela Unidade de Epidemiologia do Instituto de Medicina Preventiva e Saúde Pública da Faculdade de Medicina da Universidade de Lisboa, estando sujeitos à regulamentação legal em vigor para a proteção de dados. Não se colocam outros problemas éticos.

\section{Intervenções preventivas de comunicação em saúde (IPCS)}

As IPCS serão baseadas em metodologias de CS, tendo como público-alvo a população do Inquérito Nacional INCEAD STP. Considerar-se-ão os consumos excessivos de AD e serão desenhadas para os diferentes grupos etários. Estas intervenções, além de constituírem abordagens inovadoras envolvendo as populações alvo, adotarão metodologias científicas. Foram já ensaiadas em meio escolar no ano de 2013 algumas IPCS, designadamente com Embaixador UNICEF - corredor de Fórmula 3000 e Karts - no sentido de prevenir o CE de AD e condução de motos, e com jogadores profissionais de futebol "Os Belenenses", seguindo as recomendações do Programa das Nações Unidas para o Desenvolvimento Humano (PNUD), mobilizando, através de audiência oficial, o Chefe de Estado e Presidente da República, Manuel Pinto da Costa.

\section{População-alvo nas IPCS}

Neste projeto de investigação, pretendemos realizar intervenções preventivas, junto da população alvo do estudo, divididas por diferentes grupos etários (13 - 14; 15 - 16; 17 18; 19 - 20 e 20 - 25 anos de idade). Estas intervenções são consideradas instrumentos essenciais da Comunicação em Saúde suscetíveis de prevenir CEAD, capacitadoras da população alvo e mobilizadoras para o empoderamento em saúde.

\section{Métodos de intervenção}

As principais IPCS recorrerão a inovadoras metodologias de edutainment, seguindo o modelo da Soul City, ${ }^{55}$ desenvolvidas com recurso a diferentes modalidades desportivas, adaptadas às caraterísticas do país, aos equipamentos desportivos e território e da população-alvo. As IPCS serão ensaiadas previamente em diferentes grupos alvo - focus groups - visando a capacitação e o empoderamento dos professores de Educação Física, no seu papel de mediadores e/ou embaixadores das metodologias; a mobilização da população-alvo com a criação de mais treinos no sentido do desenvolvimento de competição desportiva. As principais IPCS poderão ainda resultar na criação de um programa de televisão e rádio, EDUCA_PRESS, com o envolvimento dos embaixadores de edutainment das diferentes IPCS em atividades de apoio desportivo ou escolar online, onde existam tecnologias para o efeito, ou com recurso aos players privados e eventos em rede social destinados a jovens da população-alvo. Pretendemos assim, através do edutainment com a CS, desenvolver atitudes e novos comportamentos que visem a promoção de estilos de vida saudáveis e práticas desportivas nos jovens. Nestas ações será feita uma articulação com o Comité Olímpico Internacional (COI) designadamente através de acordo com o Olympic Solidarity Programme. Considerando as dificuldades do território onde o projeto de investigação se desenvolve, por um lado, e, por outro, após discussão com representante de $\mathrm{COI}$, consideramos que as modalidades que se aplicam a São Tomé e Príncipe e aos géneros feminino e masculino são andebol, futebol, atletismo e natação (Fig. 2).

\section{Avaliação}

Com as intervenções preventivas de Comunicação em Saúde pretendemos a prevenção de CEAD e a redução de danos causados pelos CEAD na população alvo. Nestes termos, será feita uma avaliação dos ensaios e projetos de Comunicação em Saúde, e seu impacto na prevenção de consumos excessivos e promoção da saúde dos jovens de STP. 


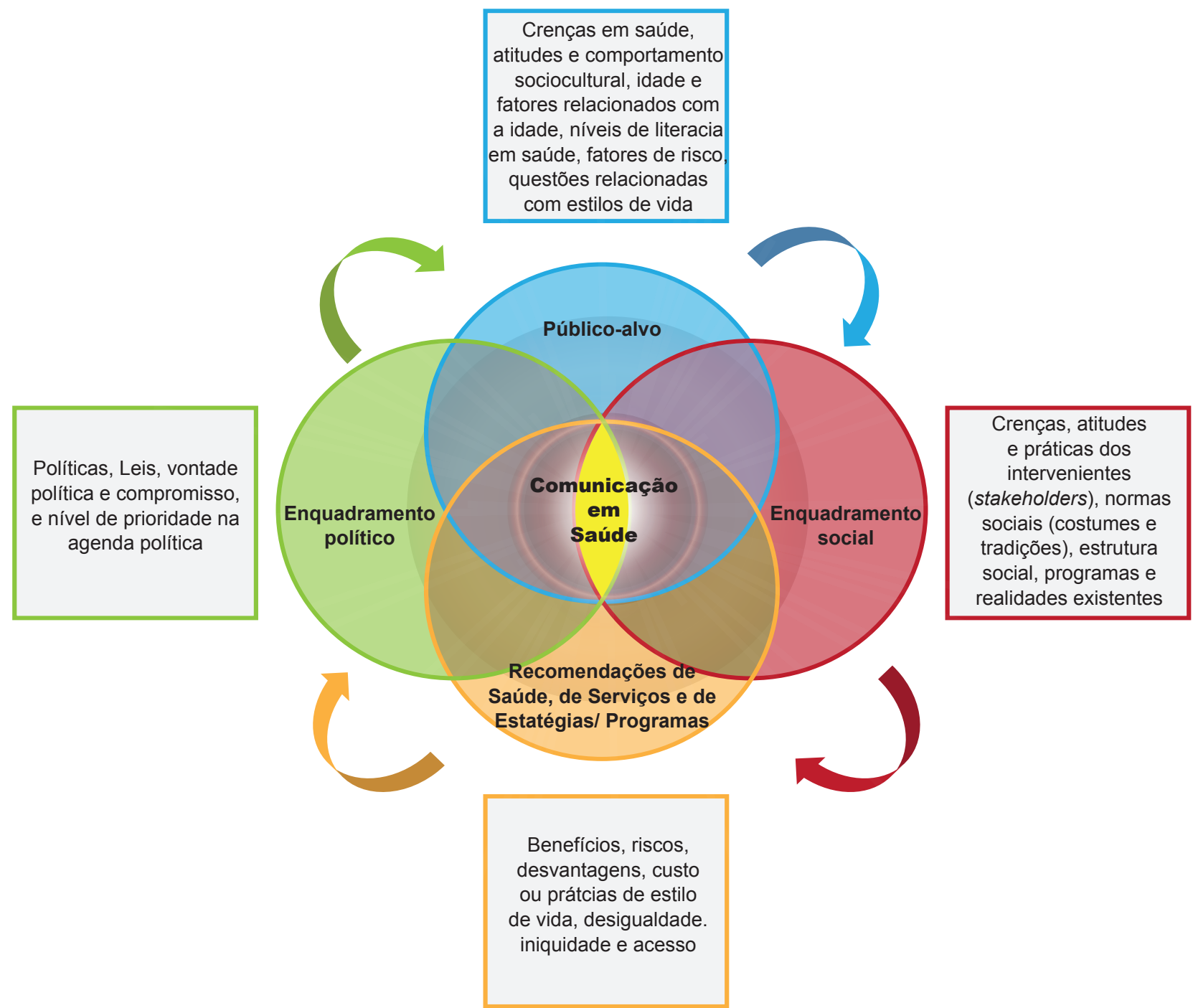

Figura 2 - Modelo de determinantes de Comunicação em Saúde Pública

\section{Cronograma}

No desenvolvimento deste estudo, dividido em três partes fundamentais, prevêem-se as atividades seguintes: recolha de dados, tratamento e análise dos resultados, exposição das conclusões e intervenções preventivas. A gestão do projeto é da responsabilidade da investigadora responsável. No decurso da aplicação dos questionários, tanto na recolha da amostra de conveniência, inquérito-piloto, como na recolha da amostra INCEAD STP prevemos uma articulação direta com o Ministério da Educação, Cultura e Formação e com a Direção Nacional das Escolas Secundárias, com vista a consultas para atualização do questionário e adaptação do questionário à linguagem utilizada pelo população-alvo. Posteriormente, pretendemos produzir artigos científicos e sugerir e aplicar conteúdos de educação e comunicação em saúde, com recurso às Ferramentas Essenciais de Saúde Pública (Essential Public Health Operations, EPHO 9), considerado seu verdadeiro determinante. As $E P H O$ e as intervenções preventivas irão sustentar-se no edutainment (educação, comunicação e entretenimento em saúde com recurso ao desporto), destinando-se às diferentes faixas etárias dos públicos-alvo. Nestas IP serão considerados o nível de conhecimento e literacia em saúde e os resultados obtidos, bem como diferentes níveis de prevenção associada ao risco e problemática de comportamento dependente, o risco/base; dependência base e risco de dependência a que correspondem, respetivamente, Prevenção universal; Prevenção seletiva e Prevenção indicativa (Universal prevention; Selective prevention e Indicated prevention). O projeto e o estudo incidirão nos domínios da prevenção universal, isto é, Promoção da Saúde e Prevenção da Doença (PSPD). Com vista a atingir estes objetivos, antecipa-se a celebração de um protocolo de colaboração entre o Ministério da Justiça e dos Direitos Humanos e a Faculdade de Medicina da Universidade de Lisboa, que reconhece a realização de Inquérito Nacional INCEAD STP. Os objetivos: a) "A cooperação entre a FMUL-IMPSP e o MJDH para que se estabeleçam as metodologias específicas nos domínios da investigação, educação e comunicação em saúde;" e b) "Medidas de intervenção - estratégias de comunicação em saúde - visando a prevenção junto do público-alvo específico, através de especialistas e 


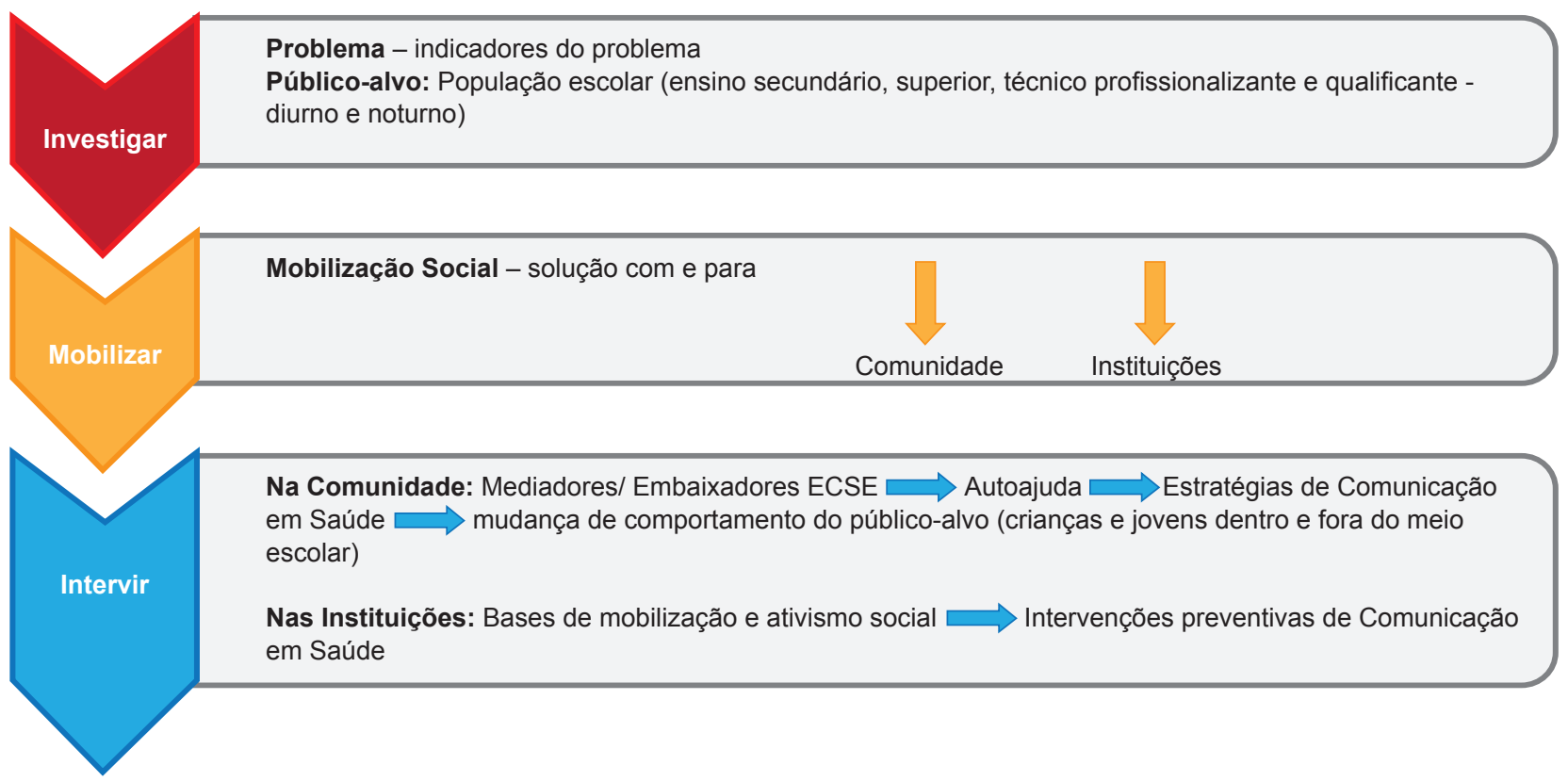

Figura 3 - Lócus de problemas - que problema de Saúde Pública e soluções - na mobilização da comunidade em STP e das instituições santomenses

Fonte: De Santiago I, 2015, Health Communication Prevention, AMP. Dearing JW, 2003. The state of the art and the state of science of community organizing

investigadores". O projeto de investigação insere-se no código 2310.0 - Professores dos ensinos universitários e superior) e tem o código 2.22. ISCO/08 - Especialistas das atividades intelectuais e científicas (profissionais de saúde), dentro da Classificação Nacional das Profissões de São Tomé e Príncipe (CNP-STP) Apêndice 1 (ver Apêndice 1: https://www.actamedicaportuguesa.com/revista/index. php/amp/article/view/13435/Apendice_01.pdf), Fig. 3.

\section{AGRADECIMENTOS}

Os autores antecipam os agradecimentos a: Diocese de São Tomé e Príncipe, Companhia de Santa Teresa de Jesus, Santa Casa de Misericórdia de São Tomé e Príncipe, Delegado de Saúde, Distrito de Lembá, Presidente da Região Autónoma de Príncipe, anterior Ministro da Educação, Cultura e Formação de São Tomé e Príncipe, atual Primeiro Ministro e anterior Ministro da Justiça e Direitos Humanos, e TAP Air Portugal, Direção Geral do Ensino Secundário e Formação, Presidente do Instituto Superior Politécnico de São Tomé e Príncipe e Homero Moreno, Professor na Direção Geral do Ensino Secundário e Formação.

Os autores agradecem ainda a Ana P. Martins, atual bastonária da Ordem dos Farmacêuticos, Paulo Cleto Duarte, presidente da Associação Nacional de Farmácias, Dario B. Martins secretário-geral da Associação de Farmácias de Língua Portuguesa e equipa do Laboratório de Estudos Farmacêuticos, respetivamente, no aconselhamento e apoio científico com vista à realização de análises de 28 amostras de álcoois artesanais das duas ilhas, pelo LEF e pelo Laboratório Nacional de Engenharia Civil (LNEC), Laboratório de Estado Português.

Os autores agradecem finalmente a revisão crítica do manuscrito realizada por Ruy M. Ribeiro.

\section{PROTEÇÃO DE PESSOAS E ANIMAIS}

Os autores declaram que os procedimentos seguidos estão de acordo com os regulamentos estabelecidos pelos responsáveis da Comissão de Investigação Clínica e Ética e de acordo com a Declaração de Helsínquia da Associação Médica Mundial.

\section{CONFIDENCIALIDADE DOS DADOS}

Os autores declaram seguir os protocolos do seu centro de trabalho acerca da publicação de dados.

\section{CONFLITOS DE INTERESSE}

Os autores declaram não possuir conflitos de interesses relacionados com o presente trabalho.

\section{FONTES DE FINANCIAMENTO}

Os custos globais do projeto, estimados em $€ 31$ 665, referentes a deslocações de cada fase do inquérito, aquisições de bens e serviços, e equipamentos (informáticos) serão, na íntegra, pagos pela investigadora responsável.

\section{REFERÊNCIAS}

1. World Health Organization. What are social determinants of health? Geneva: WHO; 2015.

2. Marmot M. The solid facts: the social determinants of health. Heal Promot J Aust Off J Aust Assoc Heal Promot Prof. 1999;9:133.
3. Wallace RB, Kohatsu N, Last JM, editors. Health disparities and health equity: concepts and measurement. In: Publich health \& preventive medicine. $15^{\text {th }}$ ed. New York: McGraw-Hill Medical; 2008.

4. Kehler DS. Addressing social inequalities for longevity and living in good 
health. Lancet Public Heal. 2020;5:E8-9.

5. Marmot M. Health in an unequal world. Lancet. 2006;368:2081-94.

6. Henschke N, Mirny A, Haafkens JA, Ramroth H, Padmawati S, Bangha $\mathrm{M}$, et al. Strengthening capacity to research the social determinants of health in low- and middle-income countries: Lessons from the INTREC programme. BMC Public Health. 2017;17:514.

7. World Health Organization. Sao Tome and Principe. Alcohol consumption: levels and patterns. 2010. [consultado 2019 Out 22] Disponível em: https://www.who.int/substance_abuse/publications/ global_alcohol_report/profiles/stp.pdf.

8. World Health Organization - Regional Office for Africa 2007. Harmful use of alcohol in the who african region: situation analysis and perspectives. Report of the Regional Director, Executive Summary. 2007. [consultado 2020 jan 19]. Disponível em: https://apps.who.int/iris/bitstream/ handle/10665/1881/AFR-RC57-14. pdf?sequence=1\&isAllowed=y.

9. Lachenmeier DW, Taylor BJ, Rehm J. Alcohol under the radar: do we have policy options regarding unrecorded alcohol? Int J Drug Policy. 2011;22:153-60.

10. António R, Marinho RT. Dossier Problemas Ligados ao Álcool. Rev Port Clin Geral. 2008;24:293-300.

11. Roswall N, Weiderpass $\mathrm{E}$. Alcohol as a risk factor for cancer: existing evidence in a global perspective. J Prev Med Public Heal. 2015;48:1-9.

12. World Health Organization. Global Status Report on noncommunicable diseases 2014. Geneva: WHO; 2014.

13. World Health Organization. Global status report on alcohol and health. Geneva: WHO; 2014.

14. George A. Global Status Report on Alcohol 2004. Can J Public Heal. 2006;97.

15. World Health Organization. Country profiles african region. Glob Status Rep Alcohol. Geneva: WHO; 2004.

16. World Health Organization. Mortality in Sao Tome and Principe. 2008:2008-2010. [consultado 2019 Oct 22]. Disponível em: http:// www.aho.afro.who.int/profiles_information/index.php/Sao_Tome_and_ Principe:Mortality/pt.

17. World Health Organization - Regional Office for Africa. Atlas of African Health Statistics 2014. Brazzaville: WHO; 2014.

18. Marques A, Demetriou Y, Tesler R, Gouveia ÉR, Peralta M, Matos MG de. Healthy lifestyle in children and adolescents and its association with subjective health complaints: findings from 37 countries and regions from the HBSC Study. Int J Environ Res Public Health. 2019;16:3292.

19. Flor LS, Gakidou E. The burden of alcohol use : better data and strong policies towards a sustainable development. Lancet Public Health. 2020:5:10-1.

20. United Nations Office on Drugs and Crime. World Drug Report 2016. New York: UN; 2016

21. Patel V, Flisher AJ, Hetrick S, McGorry P. Mental health of young people: a global public-health challenge. Lancet. 2007;369:1302-13.

22. Patel V, Thornicroft G. Packages of care for mental, neurological, and substance use disorders in low- and middle-income countries: PLOS Medicine series. PLoS Med. 2009;6: e1000160.

23. Lopez AD, Mathers CD. Measuring the global burden of disease and epidemiological transitions: 2002-2030. Ann Trop Med Parasitol. 2016;100:481-99.

24. Mathers CD, Loncar D. Projections of global mortality and burden of disease from 2002 to 2030. PLoS Med. 2006;3:2011-30.

25. Casswell S, Pledger M, Hooper R. Socioeconomic status and drinking patterns in young adults. Addiction. 2003;98:601-10.

26. Gowing LR, Ali RL, Allsop S, Marsden J, Turf EE, West R, et al. Global statistics on addictive behaviours: 2014 status report. Addiction. 2015;110:904-19.

27. Omaswa F, Crisp N, editors. African health leaders: making change and claiming the future. Oxford: Oxford University Press. 2015.

28. Vinet L, Zhedanov A. A 'missing' family of classical orthogonal polynomials. Acad Med. 2010;92:462-7.

29. Padrão $P$, Damasceno $A$, Silva-Matos $C$, Laszczyńska O, Prista A, Gouveia L, et al. Alcohol consumption in Mozambique: Regular consumption, weekly pattern and binge drinking. Drug Alcohol Depend. 2011;115:87-93.

30. United Nations Office on Drugs and Crime. World Drug Report 2018. New York: UN; 2018.

31. Nations Unies - Comission Économique pour l'Afrique. Profil de pays 2017. Sao Tomé-et-Principe. Addis-Abeba: UN; 2017.

32. Rispel LC, Palha de Sousa CA, Molomo BG. Can social inclusion policies reduce health inequalities in sub-Saharan Africa? A rapid policy appraisal. J Heal Popul Nutr. 2009;27:505-17.

33. Brooks-Gunn J, Duncan GJ. The effects of poverty on children. Futur Child. 1997;7:55-71

34. Hofman K, Blomstedt Y, Addei S, Kalage R, Maredza M, Sankoh $O$, et al. Addressing research capacity for health equity and the social determinants of health in three African countries: The INTREC programme. Glob Health Action. 2013;6:19668.

35. Barrio P, Reynolds J, García-Altés A, Gual A, Anderson P, Rehm J, et al. Social costs ofillegal drugs, alcohol and tobacco in the EuropeanUnion: a systematic review. Eur Addict Res. 2014;49:205-12.

36. World Health Organization - Regional Office for Africa. São Tomé et Principe Enquête STEPS Note de synthèse Sao Tomé et Principe Enquête STEPS Note de Synthèse. Brazzaville: WHO; 2008.

37. Casswell S, Thamarangsi T. Reducing harm from alcohol: call to action. Lancet. 2009;373:2247-57.

38. Kanteres F, Rehm J, Lachenmeier DW. Artisanal alcohol production in Mayan Guatemala: chemical safety evaluation with special regard to acetaldehyde contamination. Sci Total Environ. 2009;407:5861-8.

39. Wood AM, Kaptoge S, Butterworth AS, Willeit P, Warnakula S, Bolton T, et al. Risk thresholds for alcohol consumption: combined analysis of individual-participant data for 599912 current drinkers in 83 prospective studies. Lancet. 2018;391:1513-23.

40. Ferreira-Borges C, Esser MB, Dias S, Babor T, Parry CD. Alcohol control policies in 46 African countries: opportunities for improvement. Alcohol Alcohol. 2015;50:470-6.

41. Ferreira-Borges C, Parry CD, Babor TF. Harmful use of alcohol: a shadow over sub-Saharan Africa in need of workable solutions. Int J Environ Res Public Health. 2017;14:346.

42. World Health Organization. A framework for implementing the se $t$ of recommendations on the marketing of foods and non-alcoholic beverages to children. Geneve: WHO; 2012.

43. World Health Organization. Sao Tome and Principe Factsheet of Health Statistics 2018. Brazzaville: WHO African Health Obs. 2018:0-21.

44. Young NK. Effects of alcohol and other drugs on children. J Psychoactive Drugs. 1997;29:23-42.

45. Clausen T, Rossow I, Naidoo N, Kowal P. Diverse alcohol drinking patterns in 20 African countries. Addiction. 2009;104:1147-54.

46. Anderson P, Chisholm D, Fuhr DC. Effectiveness and cost-effectiveness of policies and programmes to reduce the harm caused by alcohol. Lancet. 2009;373:2234-46.

47. Macleod J, Oakes R, Oppenkowski T, Stokes-lampard H, Copello A, Crome I. et al. How strong is the evidence that illicit drug use by young people is an important cause of psychological or social harm? Methodological and policy implications of a systematic review of longitudinal, general population studies. Drugs Educ Prev Policy. 2004;11:281-297.

48. Casswell S. Alcohol brands in young peoples' everyday lives: new developments in marketing. Alcohol Alcohol. 2004;39:471-6.

49. Rimal RN, Lapinski MK. Why health communication is important in public health. Bull World Health Organ. 2009;87:247.

50. Soul City Institute. Edutainment: using stories \& media for social action and behaviour change. 2014. [consultado 2020 jan 19]. Disponível em: https://www.soulcity.org.za/resources/research/published-articles/ edutainment-using-stories-and-media-for-social-action-and-behaviourchange/view.

51. Reiman A. Cannabis as a substitute for alcohol and other drugs. Harm Reduct J. 2009;6:35.

52. Stockwell T, Zhao J, Panwar S, Roemer A, Naimi T, Chikritzhs T. Do "moderate" drinkers have reduced mortality risk? A systematic review and meta-analysis of alcohol consumption and all-cause mortality. J Stud Alcohol Drugs. 2016;77185-98.

53. Glanz K, Bishop DB. The role of behavioral science theory in development and implementation of public health interventions. Annu Rev Public Health. 2010;31:399-418. inserida

54. Modeste N, Tamayose TS, editors. Dictionary of Public Health Promotion and Education: terms and Concepts. $2^{\text {nd }}$ ed. New Jersey: Wiley; 2004.

55. Balsa C, Vital C, Urbano C. III Inquérito Nacional ao Consumo de Substâncias Psicoativas na População Geral, Portugal 2012. Lisboa: SICAD; 2014

56. Pinheiro A, Picanço P, Barbeiro J. A realidade do consumo de drogas nas populações escolares. Rev Port Clin Geral. 2011;27:348-55. 